\title{
A New Regularity Lemma and Faster Approximation Algorithms for Low Threshold Rank Graphs
}

\author{
Shayan Oveis Gharan* $\quad$ Luca Trevisan ${ }^{\dagger}$
}

\begin{abstract}
Kolla and Tulsiani [KT07, Kol11] and Arora, Barak and Steurer [ABS10] introduced the technique of subspace enumeration, which gives approximation algorithms for graph problems such as unique games and small set expansion; the running time of such algorithms is exponential in the threshold-rank of the graph.

Guruswami and Sinop [GS11, GS12], and Barak, Raghavendra, and Steurer [BRS11] developed an alternative approach to the design of approximation algorithms for graphs of bounded threshold-rank, based on semidefinite programming relaxations in the Lassere hierarchy and on novel rounding techniques. These algorithms are faster than the ones based on subspace enumeration and work on a broad class of problems.

In this paper we develop a third approach to the design of such algorithms. We show, constructively, that graphs of bounded threshold-rank satisfy a weak Szemeredi regularity lemma analogous to the one proved by Frieze and Kannan [FK99] for dense graphs. The existence of efficient approximation algorithms is then a consequence of the regularity lemma, as shown by Frieze and Kannan.

Applying our method to the Max Cut problem, we devise an algorithm that is faster than all previous algorithms, and is easier to describe and analyze.
\end{abstract}

\section{Introduction}

Kolla and Tulsiani [KT07, Kol11] and Arora, Barak and Steurer [ABS10] proved that the Unique Games problem can be approximated efficiently if the adjacency matrix of a graph associated with the problem has few large eigenvalues; they show that, for every optimal solution, its indicator vector is close to the subspace spanned by the eigenvectors of the large eigenvalues, and one can find a solution close to an optimal one by enumerating an $\epsilon$-net for such a subspace. Such subspace enumeration algorithm runs in time exponential in the dimension of the subspace, which is the number of large eigenvalues; such a parameter is called the threshold rank of the graph. Arora, Barak and Steurer show that the subspace enumeration algorithm can approximate other graph problems, in regular graphs, in time exponential in the threshold rank, including the Uniform Sparsest Cut problem, the Small-Set Expansion problem and the Max Cut problem ${ }^{1}$.

\footnotetext{
${ }^{*}$ Department of Management Science and Engineering, Stanford University. Supported by a Stanford Graduate Fellowship. Email:shayan@stanford.edu.

${ }^{\dagger}$ Department of Computer Science, Stanford University. This material is based upon work supported by the National Science Foundation under grant No. CCF 1017403. Email:trevisan@stanford.edu.

${ }^{1}$ The subspace enumeration algorithm does not improve the 0.878 approximation guarantee of Goemans, Williamson [GW95], but it finds a solution of approximation factor $1-O(\epsilon)$ if the optimum cuts at least $1-\epsilon$. fraction of edges.
} 
Barak, Raghavendra and Steurer [BRS11] and Guruswami and Sinop [GS11, GS12, GS13] developed an alternative approach to the design of approximation algorithms running in time exponential in the threshold rank. Their algorithms are based on solving semidefinite programming relaxations from the Lasserre hierarchy and then applying sophisticated rounding schemes. The advantage of this approach is that it is applicable to a more general class of graph problems and constraint satisfaction problems, that the approximation guarantee has a tighter dependency on the threshold used in the definition of threshold rank and that, in same cases, the algorithms have a running time of $f(k, \epsilon) \cdot n^{O(1)}$ where $k$ is the threshold rank and $1 \pm \epsilon$ is the approximation guarantee, instead of the running time of $n^{O(k)}$ which follows from an application of the subspace enumeration algorithm for constant $\epsilon$.

In this paper we introduce a third approach to designing algorithms for graphs of bounded threshold rank, which is based on proving a weak Szemeredi regularity lemma for such graphs.

The regularity lemma of Szemeredi [Sze78] states that every dense graph can be well approximated by the union of a constant number of bipartite complete subgraphs; the constant, however, has a tower-of-exponentials dependency on the quality of approximation. Frieze and Kannan [FK96, FK99] prove what they call a weak regularity lemma, showing that every dense graph can be approximated up to an error $\epsilon n^{2}$ in the cut norm by a linear combination of $O\left(1 / \epsilon^{2}\right)$ cut matrices (a cut matrix is a bipartite complete subgraph) with bounded coefficients. Frieze and Kannan also show that such an approximation can be constructed "implicitly" in time polynomial in $1 / \epsilon$ and that, for a weighted graph which is a linear combination of $\sigma$ cut matrices, several graph problems can be approximated in time $\exp (\tilde{O}(\sigma))+\operatorname{poly}(n)$ time. Combining the two facts one has a $\exp (\operatorname{poly}(1 / \epsilon))+\operatorname{poly}(n)$ time approximation algorithm for many graph problems on dense graphs.

We prove that a weak regularity lemma holds for all graphs of bounded threshold rank. Our result is a proper generalization of the weak regularity lemma of Frieze and Kannan, because dense graphs are known to have bounded threshold $\operatorname{rank}^{2}$. For a (weighted) $G=(V, E)$ with adjacency matrix $A$, and diagonal matrix of vertex degrees $D, D^{-1 / 2} A D^{-1 / 2}$ is called the normalized adjacency matrix of $G$. If the square sum of the eigenvalues of the normalized adjacency matrix outside the range $[-\epsilon / 2, \epsilon / 2]$ is equal to $k$ (in particular, if there are at most $k$ such eigenvalues), then we show that there is a linear combination of $O\left(k / \epsilon^{2}\right)$ cut matrices that approximate $A$ up to $2 \epsilon|E|$ in cut norm; furthermore, such a decomposition can be found in poly $(n, k, 1 / \epsilon)$ time. (See Theorem 2.3 below.) Our regularity lemma, combined with an improvement of the Frieze-Kannan approximation algorithm for graphs that are linear combination of cut matrices, gives us algorithms of running time $2^{\tilde{O}\left(k^{1.5} / \epsilon^{3}\right)}+\operatorname{poly}(n)$ for several graph problems on graphs of threshold rank $k$, providing an additive approximation of $2 \epsilon|E|$. In problems such as Max Cut in which the optimum is $\Omega(|E|)$, this additive approximation is equivalent to a multiplicative approximation.

\begin{tabular}{|c|c|c|}
\hline Reference & Running time & Parameter $k$ \\
[BRS11 $^{*}$ & $2^{O\left(k / \epsilon^{4}\right)} \cdot \operatorname{poly}(n)$ & \# of eigenvalues not in range $\left[-c \cdot \epsilon^{2}, c \cdot \epsilon^{2}\right], c>0$ \\
{$[\mathrm{GS} 11]$} & $n^{O\left(k / \epsilon^{2}\right)}$ & \# of eigenvalues $\leq-\epsilon / 2$ \\
[GS12] & $2^{k / \epsilon^{3}} \cdot n^{O(1 / \epsilon)}$ & \# of eigenvalues $\leq-\epsilon^{2} / 2$ \\
\hline this paper & $2^{O\left(k^{1.5} / \epsilon^{3}\right)}+$ poly $(n)$ & sum of squares of eigenvalues not in range $[-\epsilon / 8, \epsilon / 8]$ \\
\hline
\end{tabular}

Table 1: A comparison between previous algorithms applied to Max Cut and our algorithm. [BRS11] needs to solve $r$ rounds of Lasserre hierarchy, for $r=O\left(k / \epsilon^{4}\right)$.

\footnotetext{
${ }^{2}$ The normalization used for dense graphs is different.
} 
Table 1 gives a comparison between previous algorithms applied to Max Cut and our algorithm. The advantages over previous algorithms, besides the simplicity of the algorithm, is a faster running time and the dependency on a potentially smaller theshold-rank parameter, because the running time of our algorithm depends on the sum of squares of eigenvalues outside of a certain range, rather than the number of such eigenvalues. (recall that the eigenvalues of $D^{-1 / 2} A D^{-1 / 2}$ are in the range $[-1,1]$.)

We now give a precise statement of our results, after introducing some notation.

\section{Statement of Results}

\subsection{Notations}

Let $G=(V, E)$ be a (weighted) undirected graph with $n:=|V|$ vertices. Let $A$ be the adjacency matrix of $G$. For a vertex $u \in V$, let $d(u):=\sum_{v \sim u} A(u, v)$ be the degree of $u$. For a set $S \subset V$, let $d(S)=\sum_{v \in S} d(v)$ be the summation of vertex degrees in $S$, and and let $m:=2|E|=d(V)$. Let $D$ be the diagonal matrix of degrees. For any matrix $M \in \mathbb{R}^{V \times V}$, we use $M_{\mathcal{D}}$ to denote the symmetric matrix $D^{-1 / 2} M D^{-1 / 2}$. We call $A_{\mathcal{D}}$ the normalized adjacency matrix of $G$. It is straightforward to see that all eigenvalues of $A_{\mathcal{D}}$ is contained in the interval $[-1,1]$.

For two functions $f, g \in \mathbb{R}^{V}$, let $\langle f, g\rangle:=\sum_{v \in V} f(v) g(v)$. Also, let $f \otimes g$ be the tensor product of $f, g$; i.e., the matrix in $\mathbb{R}^{V \times V}$ such that $(u, v)$ entry is $f(u) g(v)$. For a function $f \in \mathbb{R}^{V}$, and $S \subseteq V$ let $f(S):=\sum_{v \in S} f(v)$.

For a set $S \subseteq V$, let $\mathbf{1}_{S}$ be the indicator function of $S$, and let

$$
d_{S}(v):= \begin{cases}d(v) & v \in S \\ 0 & \text { otherwise. }\end{cases}
$$

For any two sets $S, T \subseteq V$, and $\alpha \in \mathbb{R}$, we use the notation $\operatorname{CUT}(S, T, \alpha):=\alpha d_{S} \otimes d_{T}$ to denote the matrix corresponding to the cut $(S, T)$, where $(u, v)$ entry of the matrix is $\alpha d(u) d(v)$ if $u \in S, v \in T$ and zero otherwise. We remark that $\operatorname{CUT}(S, T, \alpha)$ is not necessarily a symmetric matrix.

Definition 2.1 (Matrix Norms). For a matrix $M \in \mathbb{R}^{V \times V}$, and $S, T \subseteq V$, let

$$
M(S, T):=\sum_{u \in S, v \in T} M_{u, v}
$$

The Frobenius norm and the cut norm are defined as follows:

$$
\begin{aligned}
\|M\|_{F} & :=\sqrt{\sum_{u, v} M_{u, v}^{2}}, \\
\|M\|_{C} & :=\max _{S, T \subseteq V}|M(S, T)|
\end{aligned}
$$

Definition 2.2 (Sum-Squares Threshold Rank). For any unweighted graph $G$, with normalized adjacency matrix $A_{\mathcal{D}}$, let $\lambda_{1}, \ldots, \lambda_{n}$ be the eigenvalues of $A_{\mathcal{D}}$ with the corresponding eigenfunctions $f_{1}, \ldots, f_{n}$. For $\delta>0$, the $\delta$ sum-squares threshold rank of $A$ is defined as

$$
t_{\delta}\left(A_{\mathcal{D}}\right):=\sum_{i:\left|\lambda_{i}\right|>\delta} \lambda_{i}^{2}
$$


Also, the $\delta$ threshold approximation of $A$ is defined as,

$$
T_{\delta}\left(A_{\mathcal{D}}\right):=\sum_{i:\left|\lambda_{i}\right|>\delta} \lambda_{i} f_{i} \otimes f_{i}
$$

\subsection{Matrix Decomposition Theorem}

The following matrix decomposition theorem is the main technical result of this paper.

Theorem 2.3. For any graph $G$, and $\epsilon>0$, let $k:=t_{\epsilon / 2}\left(A_{\mathcal{D}}\right)$. There is a algorithm that writes $A$ as a linear combination of cut matrices, $W^{(1)}, W^{(2)}, \ldots, W^{(\sigma)}$, such that $\sigma \leq 16 k / \epsilon^{2}$, and

$$
\left\|A-W^{(1)}-\ldots-W^{(\sigma)}\right\|_{C} \leq \epsilon m
$$

where each $W^{(i)}$ is a cut matrix $\operatorname{CUT}(S, T, \alpha)$, for some $S, T \subseteq V$, such that $|\alpha| \leq \sqrt{k} / m$. The running time of the algorithm is polynomial in $n, k, 1 / \epsilon$.

\subsection{Algorithmic Applications}

Our main algorithmic application of Theorem 2.3 is the following theorem that approximates any cut on low threshold rank graphs with a running time $2^{\tilde{O}\left(k^{1.5} / \epsilon^{3}\right)}+\operatorname{poly}(n)$.

Theorem 2.4. Let $G=(V, E)$, and for a given $0<\epsilon$, let $k:=t_{\epsilon / 8}\left(A_{\mathcal{D}}\right)$. There is a randomized algorithm such that for any maximization or minimization problem on sets of size $\Gamma$ in time $2^{\tilde{O}\left(k^{1.5} / \epsilon^{3}\right)}+\operatorname{poly}(n)$ finds a random set $S$ such that $|d(S)-\Gamma| \leq \epsilon m$, and with constant probability for any $S^{*}$ of size $d\left(S^{*}\right)=\Gamma$,

$$
A(S, \bar{S}) \geq A\left(S^{*}, \overline{S^{*}}\right)-\epsilon m
$$

if it is a maximization problem, otherwise,

$$
A(S, \bar{S}) \leq A\left(S^{*}, \overline{S^{*}}\right)+\epsilon m .
$$

We can use the above theorem to provide a PTAS for maximum cut, maximum bisection, and minimum bisection problems.

Corollary 2.5. Let $G=(V, E)$, and for a given $\epsilon>0$, let $k:=t_{\epsilon / 8}\left(A_{\mathcal{D}}\right)$. There is a randomized algorithm that in time $2^{\tilde{O}\left(k^{1.5} / \epsilon^{3}\right)}+\operatorname{poly}(n)$ finds an $\epsilon m$ additive approximation of the maximum cut.

Proof. We can simply guess the size of the optimum within an $\epsilon m / 2$ additive error and then use Theorem 2.4.

Corollary 2.6. Let $G=(V, E)$, and for a given $\epsilon>0$, let $k:=t_{\epsilon / 8}\left(A_{\mathcal{D}}\right)$. For any of the maximum bisection and minimum bisection problems, there is a randomized algorithm that in time $2^{\tilde{O}\left(k^{1.5} / \epsilon^{3}\right)}+\operatorname{poly}(n)$ finds a cut $(S, \bar{S})$ such that $|d(S)-m / 2| \leq \epsilon m$ and that $A(S, \bar{S})$ provides an $\epsilon m$ additive approximation of the optimum.

Proof. For the maximum/minimum bisection the optimum must have size $m / 2$. So we can simply use Theorem 2.4 with $\Gamma=m / 2$. 


\section{Regularity Lemma for Low Threshold Rank Graphs}

In this section we prove Theorem 2.3. The first step is to approximate $A$ by a low rank matrix $B$. In the next lemma we construct $B$ such that the value of any cut in $A$ is approximated within an small additive error in $B$.

Lemma 3.1. Let $A$ be the adjacency matrix of $G$. For $0 \leq \delta<1$, let

$$
B:=D^{1 / 2} T_{\delta}\left(A_{\mathcal{D}}\right) D^{1 / 2} .
$$

Then, $\|A-B\|_{C} \leq \delta m$.

Proof. Let $\lambda_{1}, \ldots, \lambda_{n}$ be the eigenvalues of $A_{\mathcal{D}}$, with the corresponding eigenfunctions $f_{1}, \ldots, f_{n}$. For any $S, T \subseteq V$, we have

$$
\begin{aligned}
\left\langle\mathbf{1}_{S},(A-B) \mathbf{1}_{T}\right\rangle & =\left\langle D^{1 / 2} \mathbf{1}_{S},(A-B)_{\mathcal{D}} D^{1 / 2} \mathbf{1}_{T}\right\rangle \\
& =\left\langle\sqrt{d_{S}},\left(A_{\mathcal{D}}-T_{\delta}\left(A_{\mathcal{D}}\right)\right) \sqrt{d_{T}}\right\rangle \\
& \leq \delta \sum_{i:\left|\lambda_{i}\right| \leq \delta}\left\langle\sqrt{d_{S}}, f_{i}\right\rangle\left\langle\sqrt{d_{T}}, f_{i}\right\rangle \\
& \leq \delta \sqrt{\sum_{i:\left|\lambda_{i}\right| \leq \delta}\left\langle\sqrt{d_{S}}, \mathbf{x}_{i}\right\rangle^{2}} \cdot \sqrt{\sum_{i:\left|\lambda_{i}\right| \leq \delta}\left\langle\sqrt{d_{T}}, f_{i}\right\rangle^{2}} \\
& \leq \delta\left\|\sqrt{d_{S}}\right\|\left\|\sqrt{d_{T}}\right\| \leq \delta\left\|\sqrt{d_{V}}\right\|^{2}=\delta m,
\end{aligned}
$$

where the second inequality follows by the Cauchy-Schwarz inequality. The lemma follows by noting the fact that $\|A-B\|_{C}$ is the maximum of the above expression for any $S, T \subseteq V$.

By the above lemma if we approximate $B$ by a linear combination of cut matrices, that also is a good approximation of $A$. Moreover, since $t_{\delta}\left(A_{\mathcal{D}}\right)=t_{\delta}\left(B_{\mathcal{D}}\right), B$ has a small sum-square threshold rank iff $A$ has a small threshold rank.

Lemma 3.2. For any graph $G$ with adjacency matrix $A$, and $\delta>0$, let $B:=D^{1 / 2} T_{\delta}\left(A_{\mathcal{D}}\right) D^{1 / 2}$. Then,

$$
\left\|B_{\mathcal{D}}\right\|_{F}^{2}=t_{\delta}\left(A_{\mathcal{D}}\right)
$$

Proof. The lemma follows from the fact that the frobenius norm of any matrix is equal to the summation of square of eigenvalues. If $\lambda_{1}, \ldots, \lambda_{n}$ are the eigenvalues of $A_{\mathcal{D}}$, then

$$
\|B\|_{F}^{2}=\operatorname{trace} B^{2}=\sum_{\left|\lambda_{i}\right|>\delta} \lambda_{i}^{2}=t_{\delta}\left(A_{\mathcal{D}}\right) .
$$

The next proposition is the main technical part of the proof of Theorem 2.3. We show that we can write any (not necessarily symmetric) matrix $B$ as a linear combination of $O\left(\|B\|_{F}^{2} / \epsilon^{2}\right)$ cut matrices such that the cut norm of $B$ is preserved within an additive error of $\epsilon m$. The proof builds on the existential theorem of Frieze and Kanan [FK99, Theorem 7]. 
Proposition 3.3. For any matrix $B \in \mathbb{R}^{V \times V}, k=\left\|B_{\mathcal{D}}\right\|_{F}^{2}$, and $\epsilon>0$, there exist cut matrices $W^{(1)}, W^{(2)}, \ldots, W^{(\sigma)}$, such that $\sigma \leq 1 / \epsilon^{2}$, and for all $S, T \subseteq V$,

$$
\left|\left(B-W^{(1)}-W^{(2)}-\ldots-W^{(\sigma)}\right)(S, T)\right| \leq \epsilon \sqrt{k} \sqrt{d(S) d(T)},
$$

where each $W^{(i)}$ is a cut matrix $\operatorname{CUT}(S, T, \alpha)$, for some $S, T \subseteq V$, and $\alpha \in \mathbb{R}$.

Proof. Let $R^{(0)}=B$. We use the potential function $h(R):=\left\|R_{\mathcal{D}}\right\|_{F}$. We show that while $\|R\|_{C}>$ $\epsilon \sqrt{k} m$, we can choose cut matrices iteratively while maintaining the invariant that each time the value of the potential function decreases by at least $\epsilon^{2} h(B)$. Since $h\left(R^{(0)}\right)=h(B)$, after at most $1 / \epsilon^{2}$ we obtain a good approximation of $B$.

Assume that after $t<1 / \epsilon^{2}$ iterations, $R^{(i)}=B-W^{(1)}-\ldots-W^{(i)}$. Suppose for some $S, T \subseteq V$,

$$
\left|R^{(i)}(S, T)\right|>\epsilon h(B) \sqrt{d(S) d(T)} .
$$

Choose $W^{(i+1)}=\operatorname{CUT}(S, T, \alpha)$, for $\alpha=R^{(i)}(S, T) / d(S) d(T)$, and let $R^{(i+1)}=R^{(i)}-W^{(i+1)}$. We have,

$$
\begin{aligned}
h\left(R^{(i+1)}\right)-h\left(R^{(i)}\right)=\sum_{u \in S, v \in T} \frac{\left(R_{u, v}^{(i)}-\alpha d(u) d(v)\right)^{2}-R_{u, v}^{(i)}{ }^{2}}{d(u) d(v)} & =-2 \alpha R^{(i)}(S, T)+\alpha^{2} d(S) d(T) \\
& =\frac{-R^{(i)}(S, T)^{2}}{d(S) d(T)} \leq-\epsilon^{2} \Phi^{2}(B),
\end{aligned}
$$

where the second to last equation follows from the definition of $\alpha$, and the last equation follows from equation (1). Therefore, after at most $\sigma \leq 1 / \epsilon^{2}$ iterations, (1) must hold for all $S, T \subseteq V$.

Although the previous proposition only proves the existence of a decomposition into cut matrices, we can construct such a decomposition efficiently using the following nice result of Alon and Naor [AN06] that gives a consant factor approximation algorithm for the cut norm of any matrix.

Theorem 3.4 (Alon and Naor [AN06]). There is a polynomial time randomized algorithm such that for any given $A \in \mathbb{R}^{V \times V}$, with high probability, finds sets $S, T \subseteq V$, such that

$$
|A(S, T)| \geq 0.56\|A\|_{C}
$$

Now we are ready to prove Theorem 2.3.

Proof of Theorem 2.3. Let $\delta:=\epsilon / 2$, and $B:=D^{1 / 2} T_{\delta}\left(A_{\mathcal{D}}\right) D^{1 / 2}$. By Lemma 3.1, we have that $\|A-B\|_{C} \leq \epsilon m / 2$. So we just need to approximate $B$ by a set of cut matrices within an additive error of $\epsilon m / 2$. For a matrix $R$, let $h(R):=\left\|R_{\mathcal{D}}\right\|_{F}$. By Lemma 3.2 we have $h(B)=\sqrt{k}$.

Let $\epsilon^{\prime}:=\epsilon / \sqrt{4 k}$. We use the proof strategy of Proposition 3.3. Let $R^{(i)}=B-W^{(1)}-\ldots-W^{(i)}$. If $\left\|R^{(i)}\right\|_{C} \geq \epsilon^{\prime} \sqrt{k} m$, then by Theorem 3.4 in polynomial time we can find $S, T \subseteq V$ such that

$$
\left|R^{(i)}(S, T)\right| \geq \epsilon^{\prime} \sqrt{k} m / 2 \geq \epsilon^{\prime} h(B) m / 2 .
$$

Choose $W^{(i+1)}=\operatorname{CUT}(S, T, \alpha)$, for $\alpha=R^{(i)}(S, T) / m^{2}$, and let $R^{(i+1)}=R^{(i)}-W^{(i+1)}$. We get,

$$
h\left(R^{(i+1)}\right)-h\left(R^{(i)}\right)=-2 \alpha R^{(i)}(S, T)+\alpha^{2} d(S) d(T) \leq-\frac{R^{(i)}(S, T)^{2}}{m^{2}} \leq-\frac{\epsilon^{\prime 2} \Phi^{2}(B)}{4} .
$$


Since $h\left(R^{(0)}\right)=h(B)$, after $\sigma \leq 4 / \epsilon^{\prime 2}=16 k / \epsilon^{2}$, we have $\left\|R^{(\sigma)}\right\|_{C} \leq \epsilon^{\prime} \sqrt{k} m$, which implies that

$$
\left\|A-W^{(1)}-\ldots-W^{(\sigma)}\right\|_{C} \leq\|A-B\|_{C}+\left\|B-W^{(1)}-\ldots-W^{(\sigma)}\right\|_{C} \leq \epsilon m .
$$

This proves the correctness of the algorithm. It remains to upper bound $\alpha$. For each cut matrix $W^{(i)}=\operatorname{CUT}(S, T, \alpha)$ constructed throughout the algorithm we have

$$
\begin{aligned}
|\alpha|=\frac{\left|R^{(i)}(S, T)\right|}{m^{2}}=\frac{1}{m^{2}}\left|\sum_{u \in S, v \in T} R_{u, v}^{(i)} \frac{\sqrt{d(u) d(v)}}{\sqrt{d(u) d(v)}}\right| & \leq \frac{1}{m^{2}} \sqrt{\sum_{u \in S, v \in T} \frac{R_{u, v}^{(i)}{ }^{2}}{d(u) d(v)}} \sqrt{d(S) d(T)} \\
& \leq \frac{h\left(R^{(i)}\right)}{m} \leq \frac{h(B)}{m}=\frac{\sqrt{k}}{m} .
\end{aligned}
$$

where the first inequality follows by the Cauchy-Schwarz inequality, the second inequality uses $d(S), d(T) \leq m$, and the last inequality follows by the fact that the potential function is decreasing throughout the algorithm. This completes the proof of theorem.

\section{Fast Approximation Algorithm for Low Threshold Rank Graphs}

In this section we prove Theorem 2.4. First, by Theorem 2.3 in time poly $(n, 1 / \epsilon)$ we can find cut matrices $W^{(1)}, \ldots, W^{(\sigma)}$ for $\sigma=O\left(k / \epsilon^{2}\right)$, such that for all $1 \leq i \leq t, W^{(i)}=\operatorname{CUT}\left(S_{i}, T_{i}, \alpha_{i}\right)$, $\alpha_{i} \leq \sqrt{k} / m$, and

$$
\|A-W\|_{C} \leq \epsilon m / 4
$$

where $W:=W^{(1)}+\ldots+W^{(\sigma)}$. It follows from the above equation that for any set $S \subseteq V$,

$$
|A(S, \bar{S})-W(S, \bar{S})|=\left|A(S, \bar{S})-\sum_{i=1}^{\sigma} \alpha_{i} d\left(S \cap S_{i}\right) d\left(\bar{S} \cap T_{i}\right)\right| \leq \frac{\epsilon m}{4} .
$$

Fix $S^{*} \subseteq V$ of size $d\left(S^{*}\right)=\Gamma$ (think of $\left(S^{*}, \overline{S^{*}}\right)$ as the optimum cut), and let $s_{i}^{*}:=d\left(S_{i} \cap S^{*}\right)$, and $t_{i}^{*}:=d\left(T_{i} \cap \overline{S^{*}}\right)$. Observe that by equation (3),

$$
\left|A\left(S^{*}, \overline{S^{*}}\right)-\sum_{i=1}^{\sigma} \alpha_{i} s_{i}^{*} t_{i}^{*}\right| \leq \frac{\epsilon m}{4} .
$$

Let $\alpha_{\max }:=\max _{1 \leq i \leq \sigma}\left|\alpha_{i}\right|$. Let $\Delta:=\left\lfloor\epsilon /\left(48 \alpha_{\max } \sigma\right)\right\rfloor$; observe that $\Delta=O\left(\epsilon^{3} m / k^{1.5}\right)$. We define an approximation of $s_{i}^{*}, t_{i}^{*}$ by rounding them down to the nearest multiple of $\Delta$, i.e., $\tilde{s}_{i}^{*}=\Delta\left\lfloor s_{i}^{*} / \Delta\right\rfloor$, and $\tilde{t}_{i}^{*}=\Delta\left\lfloor t_{i}^{*} / \Delta\right\rfloor$. We use $\tilde{s}^{*}, \tilde{t}^{*}$ to denote the vectors of the approximate values. It follows that we can obtain a good approximation of the size of the cut $\left(S^{*}, \overline{S^{*}}\right)$ just by guessing the vectors $\tilde{s}^{*}$, and $\tilde{t}^{*}$. Since $\left|s_{i}^{*}-\tilde{s}_{i}^{*}\right| \leq \Delta$ and $\left|t_{i}^{*}-\tilde{t}_{i}^{*}\right| \leq \Delta$, we get,

$$
\sum_{i=1}^{\sigma}\left|s_{i}^{*} t_{i}^{*} \alpha_{i}-\tilde{s}_{i}^{*} \tilde{t}_{i}^{*} \alpha_{i}\right| \leq \sigma \alpha_{\max }\left(2 \Delta m+\Delta^{2}\right) \leq 3 \alpha_{\max } \sigma \Delta \leq \epsilon m / 16 .
$$

Observe that by equations $(3),(4),(5)$, if we know the vectors $\tilde{s}^{*}, \tilde{t}^{*}$, then we can find $A\left(S^{*}, \overline{S^{*}}\right)$ within an additive error of $\epsilon m / 2$. Since $\tilde{s}_{i}^{*}, \tilde{t}_{i}^{*} \leq m$, there are only $O(m / \Delta)$ possibilities for each $\tilde{s}_{i}^{*}$ 
and $\tilde{t}_{i}^{*}$. Therefore, we afford to enumerate all possible values of them in time $(\mathrm{m} / \Delta)^{2 \sigma}$, and choose the one that gives the largest cut. Unfortunately, for a given assignment of $\tilde{s}^{*}, \tilde{t}^{*}$ the corresponding cut $\left(S^{*}, \overline{S^{*}}\right)$ may not exist. Next we give an algorithm that for a given assignment of $\tilde{s}^{*}, \tilde{t}^{*}$ finds a cut $(S, \bar{S})$ such that $A(S, \bar{S})=\sum_{i} \tilde{s}_{i}^{*} \tilde{t}_{i}^{*} \alpha_{i} \pm \epsilon m$, if one exists.

First we distinguish the large degree vertices of $G$ and simply guess which side they are mapped to in the optimum cut. For the rest of the vertices we use the solution of $\operatorname{LP}(1)$. Let $U:=\{v$ : $d(v) \geq \Delta\}$ be the set of large degree vertices. Observe that $|U| \leq m / \Delta$. Let $\mathcal{P}$ be the coarsest partition of the set $V \backslash U$ such that for any $1 \leq i \leq \sigma$, both $S_{i} \backslash U$ and $T_{i} \backslash U$ can be written as a union of sets in $\mathcal{P}$, and for each $P \in \mathcal{P}, d(P) \leq \Delta$. Observe that $|\mathcal{P}| \leq 2^{2 \sigma}+m / \Delta$. For a given assignment of $\tilde{s}^{*}, \tilde{t}^{*}$, first we guess the set of vertices in $U$ that are contained in $S^{*}, U_{S^{*}}:=S^{*} \cap U$, and $U_{\overline{S^{*}}}:=U \backslash U_{S^{*}}$. For the rest of the vertices we use the linear program LP(1) to find the unknown $d\left(S^{*} \cap P\right)$.

$$
\begin{array}{llll}
\operatorname{LP}(1) & \leq y_{P} & \leq 1 & \forall P \in \mathcal{P} \\
0 & & \leq \Gamma+\epsilon m / 2 & \\
\Gamma-\epsilon m / 2 & \leq \sum_{P} y_{P} d(P)+d\left(U_{S^{*}}\right) & \leq \tilde{s}_{i}^{*}+\Delta & \forall 1 \leq i \leq \sigma \\
\tilde{s}_{i}^{*} & \leq \sum_{P \subseteq S_{i}} y_{P} d(P)+d\left(U_{S^{*}} \cap S_{i}\right) & & \\
\tilde{t}_{i}^{*} & \leq \sum_{P \subseteq T_{i}}\left(1-y_{P}\right) d(P)+d\left(U_{\left.\overline{S^{*}} \cap T_{i}\right)}\right. & \leq \tilde{t}_{i}^{*}+\Delta & \forall 1 \leq i \leq \sigma .
\end{array}
$$

Observe that $y_{P}=\frac{d\left(S^{*} \cap P\right)}{d(P)}$ is a feasible solution to the linear program. In the next lemma which is the main technical part of the analysis we show how to construct a set based on a given solution of the LP.

Lemma 4.1. There is a randomized algorithm such that for any $S^{*} \subset V$, given $\tilde{s}_{i}^{*}, \tilde{t}_{i}^{*}$ and $U_{S^{*}}$ returns a random set $S$ such that

$$
\begin{aligned}
& \mathbb{P}\left[W(S, \bar{S}) \geq A\left(S^{*}, \overline{S^{*}}\right)-\frac{3 \epsilon m}{4} \wedge|d(S)-\Gamma| \leq \epsilon m\right] \geq \frac{\epsilon}{10} \\
& \mathbb{P}\left[W(S, \bar{S}) \leq A\left(S^{*}, \overline{S^{*}}\right)+\frac{3 \epsilon m}{4} \wedge|d(S)-\Gamma| \leq \epsilon m\right] \geq \frac{\epsilon}{10} .
\end{aligned}
$$

Proof. Let $y$ be a feasible solution of $\mathrm{LP}(1)$. We use a simple independent rounding scheme to compute the random set $S$. We always include $U_{S^{*}}$ in $S$. For each $P \in \mathcal{P}$, we include $P$ in $S$, independently, with probability $y_{P}$. We prove that $S$ satisfies lemma's statements. First of all, by linearity of expectation,

$$
\mathbb{E}\left[d\left(S \cap S_{i}\right)\right]=d\left(U_{S^{*}}\right)+\sum_{P \subseteq S_{i}} y_{P} d(P), \quad \text { and } \quad \mathbb{E}\left[d\left(\bar{S} \cap T_{i}\right)\right]=d\left(U_{\overline{S^{*}}}\right)+\sum_{P \subseteq T_{i}}\left(1-y_{P}\right) d(P) .
$$

In the following two claims, first we show that with high probability the expected size of $d(S)$ is close to $\Gamma$. Then, we upper bound the expected value of $W(S, \bar{S})-A\left(S^{*}, \overline{S^{*}}\right)$.

\section{Claim 4.2.}

$$
\mathbb{P}\left[\left|d(S)-d\left(S^{*}\right)\right| \geq \epsilon m\right] \leq \frac{\epsilon}{8}
$$


Proof. We use the theorem of Hoeffding to prove the claim:

Theorem 4.3 (Hoeffding Inequality). Let $X_{1}, \ldots, X_{n}$ be independent random variables such that for each $1 \leq i \leq n, X_{i} \in\left[0, a_{i}\right]$. Let $X:=\sum_{i=1}^{n} X_{i}$. Then, for any $\epsilon>0$

$$
\mathbb{P}[|X-\mathbb{E}[X]| \geq \epsilon] \leq 2 \exp \left(-\frac{2 \epsilon^{2}}{\sum_{i=1}^{n} a_{i}^{2}}\right) .
$$

Now, by the independent rounding procedure, we obtain

$\mathbb{P}[|d(S)-\mathbb{E}[d(S)]| \geq \epsilon m / 2] \leq 2 \exp \left(-\frac{\epsilon^{2} m^{2}}{2 \sum_{P} d(P)^{2}}\right) \leq 2 \exp \left(-\frac{\epsilon^{2} m^{2}}{2 m \Delta}\right) \leq 2 \exp (-16 / \epsilon) \leq \frac{\epsilon}{8}$.

where the third inequality follows by the fact that $d(P) \leq \Delta$ and $\sum_{P} d(P) \leq m$. The claim follows from the fact that by $(6),|\mathbb{E}[d(S)]-\Gamma| \leq \epsilon m / 2$.

\section{Claim 4.4.}

$$
\left|\mathbb{E}[W(S, \bar{S})]-A\left(S^{*}, \overline{S^{*}}\right)\right| \leq \frac{\epsilon m}{2}
$$

Proof. First, observe that

$$
\begin{aligned}
\mathbb{E}[W(S, \bar{S})]= & \mathbb{E}\left[\sum_{i=1}^{\sigma} d\left(S \cap S_{i}\right) d\left(\bar{S} \cap T_{i}\right) \alpha_{i}\right] \\
= & \sum_{i=1}^{\sigma} \alpha_{i} \mathbb{E}\left[\left(\sum_{P \in \mathcal{P}: P \subseteq S_{i}} d(P) \mathbb{I}[P \subseteq S]\right)\left(\sum_{Q \in \mathcal{P}: Q \subseteq T_{i}} d(Q) \mathbb{I}[Q \subseteq \bar{S}]\right)\right] \\
& +\sum_{i=1}^{\sigma} \alpha_{i}\left[d\left(U_{S^{*}} \cap S_{i}\right) \mathbb{E}\left[d\left(\bar{S} \cap T_{i}\right)\right]+d\left(U_{\overline{S^{*}}} \cap T_{i}\right) \mathbb{E}\left[d\left(S \cap S_{i}\right)\right]\right] \\
= & \sum_{i=1}^{\sigma} \alpha_{i} \sum_{P \subseteq S_{i}, Q \subseteq T_{i}} d(P) d(Q) \mathbb{E}[\mathbb{I}[P \subseteq S] \mathbb{I}[Q \subseteq \bar{S}]] \\
& +\sum_{i=1}^{\sigma} \alpha_{i}\left\{d\left(U_{S^{*}} \cap S_{i}\right) t_{i}+d\left(U_{\overline{S^{*}}} \cap T_{i}\right) s_{i}\right\}
\end{aligned}
$$

Since the event that $P \subseteq S$ is independent of $Q \subseteq S$, iff $P \neq Q$ we get

$$
\mathbb{E}[\mathbb{I}[P \subseteq S] \mathbb{I}[Q \subseteq \bar{S}]]= \begin{cases}y_{P}\left(1-y_{Q}\right) & \text { if } P \neq Q \\ 0 & \text { otherwise }\end{cases}
$$

Let $s_{i}:=\mathbb{E}\left[d\left(S \cap S_{i}\right)\right]$ and $t_{i}:=\mathbb{E}\left[d\left(\bar{S} \cap T_{i}\right)\right]$. Then, by (11) and above equation,

$$
\mathbb{E}[W(S, \bar{S})]=\sum_{i=1}^{\sigma} \alpha_{i} s_{i} t_{i}-\sum_{i=1}^{\sigma} \alpha_{i} \sum_{P \in \mathcal{P}} y_{P}\left(1-y_{P}\right) d(P)^{2}
$$

On the other hand, by equations (7) and (8), for all $1 \leq i \leq \sigma$, we get $\tilde{s}_{i}^{*} \leq s_{i} \leq \tilde{s}_{i}^{*}+\Delta$ and $\tilde{t}_{i}^{*} \leq t_{i} \leq \tilde{t}_{i}^{*}+\Delta$. Hence, similar to equation (5) we can show,

$$
\sum_{i=1}^{\sigma}\left|\alpha_{i} s_{i} t_{i}-\alpha_{i} \tilde{s}_{i}^{*} \tilde{t}_{i}^{*}\right| \leq \frac{\epsilon m}{8}
$$


Therefore, using equation (4) we get

$$
\begin{aligned}
\left|\mathbb{E}[W(S, \bar{S})]-A\left(S^{*}, \overline{S^{*}}\right)\right| & \left.\leq \mid \mathbb{E}[W(S, \bar{S})]-\sum_{i=1}^{\sigma} \alpha_{i} s_{i}^{*} t_{i}^{*}\right) \mid+\frac{\epsilon m}{4} \\
& =\left|\sum_{i=1}^{\sigma}\left(\alpha_{i} s_{i} t_{i}-\alpha_{i} s_{i}^{*} t_{i}^{*}\right)-\sum_{i=1}^{\sigma} \sum_{P \in \mathcal{P}} \alpha_{i} y_{P}\left(1-y_{P}\right) d(P)^{2}\right|+\frac{\epsilon m}{4} \\
& \leq \sum_{i=1}^{\sigma}\left|\alpha_{i} s_{i} t_{i}-\alpha_{i} \tilde{s}_{i}^{*} \tilde{t}_{i}^{*}\right|+\sum_{i=1}^{\sigma}\left|\alpha_{i} \tilde{s}_{i}^{*} \tilde{t}_{i}^{*}-\alpha_{i} s_{i}^{*} t_{i}^{*}\right|+\sigma \alpha_{\max } m \Delta+\frac{\epsilon m}{4} \leq \frac{\epsilon m}{2},
\end{aligned}
$$

where the equality follows by (12), the second inequality follows by the fact that $d(P) \leq \Delta$ for all $P \in \mathcal{P}$ and $\sum_{P} d(P) \leq m$, and the last inequality follows by equations (13) and (5). This proves the claim.

Now we are ready finish the proof of Lemma 4.1. Here, we prove (9). Equation (10) can be proved similarly. By Claim 4.4,

$$
\begin{aligned}
A\left(S^{*}, \overline{S^{*}}\right)-\frac{\epsilon m}{2} \leq \mathbb{E}[W(S, \bar{S})] & \leq \mathbb{E}[W(S, \bar{S})|| d(S)-\Gamma \mid \leq \epsilon m]+m \mathbb{P}[|d(S)-\Gamma|>\epsilon m] \\
& \leq \mathbb{E}[W(S, \bar{S})|| d(S)-\Gamma \mid \leq \epsilon m]+\frac{\epsilon m}{8}
\end{aligned}
$$

where the second inequality holds by the fact that the size of any cut in $G$ is at most $m / 2$, thus by (3) for any $S \subseteq V, W(S, \bar{S}) \leq \epsilon m / 4+m / 2 \leq m$, and the last inequality follows by Claim 4.2. Hence,

$$
\mathbb{E}[W(S, \bar{S})|| d(S)-\Gamma \mid \leq \epsilon m] \geq A\left(S^{*}, \overline{S^{*}}\right)-\frac{5 \epsilon m}{8}
$$

Since $W(S, \bar{S}) \leq m$,

$$
\mathbb{P}\left[W(S, \bar{S}) \geq A\left(S^{*}, \overline{S^{*}}\right)-\frac{3 \epsilon m}{4}|| d(S)-\Gamma \mid \leq \epsilon m\right] \geq \frac{\epsilon}{8}
$$

Therefore, (9) follows by an application of Claim 4.2.

Our rounding algorithm is described in Algorithm 1. First, we prove the correctness, then we calculate the running time of the algorithm. Let $S$ be the output set of the algorithm. First, observe that we always have $|d(S)-\Gamma| \leq \epsilon m$. Now let $A\left(S^{*}, \overline{S^{*}}\right)$ be the maximum cut among all sets of size $\Gamma$ (the minimization case can be proved similarly). In the iteration that the algorithm correctly guesses $\tilde{s}_{i}^{*}, \tilde{t}_{i}^{*}, U_{S^{*}}$, there exists a feasible solution $y$ of $\operatorname{LP}(1)$. by Lemma 4.1 , for all $1 \leq i \leq 10 / \epsilon$,

$$
\mathbb{P}\left[W\left(R_{y}(i), \overline{R_{y}(i)}\right) \geq A\left(S^{*}, \overline{S^{*}}\right)-\frac{3 \epsilon m}{4} \wedge\left|d\left(R_{y}(i)\right)-\Gamma\right| \leq \epsilon m\right] \geq \frac{\epsilon}{10}
$$

Since we take the best of $10 / \epsilon$ samples, with probability $1 / e$ the output set $S$ satisfies $W(S, \bar{S}) \geq$ $A\left(S^{*}, \overline{S^{*}}\right)-3 \epsilon m / 4$. Therefore, by $(3), A(S, \bar{S}) \geq A\left(S^{*}, \overline{S^{*}}\right)-\epsilon m$. This proves the correctness of the algorithm.

It remains to upper-bound the running time of the algorithm. First observe that if $|U|=$ $O\left(k / \epsilon^{2}\right)$, the running time of the algorithm is dominated by the time it takes to compute a feasible 


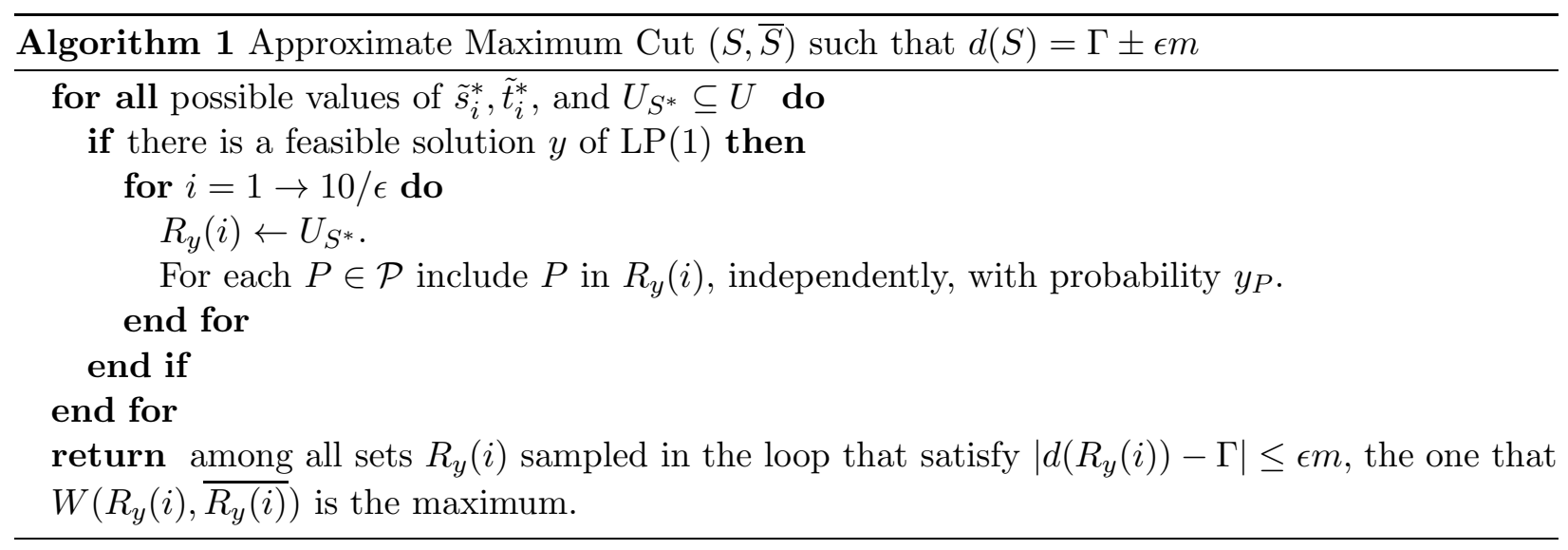

solution of $\operatorname{LP}(1)$. Since the size of LP is $2^{\tilde{O}\left(k / \epsilon^{2}\right)}$, in this case Algorithm 1 terminates in time $2^{\tilde{O}\left(k / \epsilon^{2}\right)}$. Note that for any sample set $R_{y}(i)$, both $d\left(R_{y}(i)\right)$ and $W\left(R_{y}(i), \overline{R_{y}(i)}\right)$ can be computed in time $2^{\tilde{O}\left(k / \epsilon^{2}\right)}$, once we know $\left|R_{y}(i) \cap P\right|$ for any $P \in \mathcal{P}$.

Otherwise if $|U| \gg k / \epsilon^{2}$, the dependency of the running time of the algorithm to $\epsilon, k$ is dominated by the step where we guess the subset of $U_{S^{*}}=U \cap S^{*}$. Since $\alpha_{\max } \leq \sqrt{k} / m$ and $\sigma=O\left(k / \epsilon^{2}\right)$, we get

$$
|U| \leq \frac{m}{\Delta} \leq \frac{12 m \alpha_{\max } \sigma}{\epsilon}=O\left(\frac{k^{1.5}}{\epsilon^{3}}\right) .
$$

Therefore, Algorithm 1 runs in time $2^{\tilde{O}\left(k^{1.5} / \epsilon^{3}\right)}$. Since it takes poly $(n, k, 1 / \epsilon)$ to compute the decomposition into $W^{(1)}, \ldots, W^{(\sigma)}$, the the total running time is $2^{\tilde{O}\left(k^{1.5} / \epsilon^{3}\right)}+\operatorname{poly}(n)$. This completes the proof of Theorem 2.4.

\section{References}

[ABS10] Sanjeev Arora, Boaz Barak, and David Steurer. Subexponential algorithms for unique games and related problems. In FOCS, pages 563-572, 2010. 1

[AN06] Noga Alon and Assaf Naor. Approximating the cut-norm via grothendieck's inequality. SIAM J. Comput., 35(4):787-803, April 2006. 6

[BRS11] Boaz Barak, Prasad Raghavendra, and David Steurer. Rounding semidefinite programming hierarchies via global correlation. In FOCS, pages 472-481, 2011. 1, 2

[FK96] Alan M. Frieze and Ravi Kannan. The regularity lemma and approximation schemes for dense problems. In FOCS, pages 12-, Washington, DC, USA, 1996. IEEE Computer Society. 2

[FK99] Alan M. Frieze and Ravi Kannan. Quick approximation to matrices and applications. Combinatorica, 19(2):175-220, 1999. 1, 2, 5

[GS11] Venkatesan Guruswami and Ali Kemal Sinop. Lasserre hierarchy, higher eigenvalues, and approximation schemes for graph partitioning and quadratic integer programming with psd objectives. In FOCS, pages 482-491, 2011. 1, 2 
[GS12] Venkatesan Guruswami and Ali Kemal Sinop. Faster sdp hierarchy solvers for local rounding algorithms. In FOCS, 2012. 1, 2

[GS13] Venkatesan Guruswami and Ali Kemal Sinop. Lasserre sdps, 1_1-embeddings, and approximating non-uniform sparsest cut via generalized spectra. In SODA, 2013. 2

[GW95] Michel X. Goemans and David P. Williamson. Improved approximation algorithms for maximum cut and satisfiability problems using semidefinite programming. J. ACM, 42(6):1115-1145, November 1995. 1

[Kol11] Alexandra Kolla. Spectral algorithms for unique games. Comput. Complex., 20(2):177206, June 2011. 1

[KT07] Alexandra Kolla and Madhur Tulsiani. Playing unique games using graph spectra. manuscript, 2007. 1

[Sze78] Endre Szemerdi. Regular partitions of graphs. In Problmes Combinatoires et Thorie des Graphes, volume 260 of Colloq. Internat. CNRS, pages 399-401. CNRS, Paris, 1978. 2 